\title{
A Study of the generation of laser soliton from spontaneous emission by ring cavity with carbon nanotube
}

\author{
Younis Mohamed Atiah Al-Zahy
}

College of Dentistry, Misan University, Iraq

E-mail: younisal_zahy72@yahoo.co.uk

\begin{abstract}
We demonstrate a behavior of laser pulse grows through fiber laser inside and output cavity with a soliton fiber laser based on the multi-wall carbon nanotube saturable absorber (SA), we investigate the effects of a saturable absorber parameter on the modelocking of a realistic Erbium fiber ring laser. Generalized nonlinear Schrodinger equation including the nonlinear effects as gain dispersion, second anomalous group velocity dispersion (GVD), self phase modulation (SPM), and two photon absorption used to describe pulse evolution. An analytical method has been used to understand and to quantify the role of the SA parameter on the propagation dynamics of pulse laser. We compute the chirp, power, width and phase of the soliton for range of SA parameters and we studied stability against nonlinear effects at different SA parameter ranges.
\end{abstract}

Key words

Nonlinear optics, Fibers laser, Passivemode lucking, Nanotube saturable absorber.

Article info.

Received: May. 2014

Accepted: Jun. 2014

Published: Dec. 2014

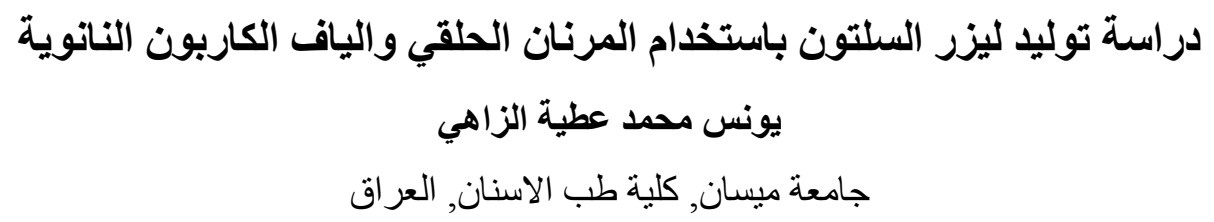

الخلاصة

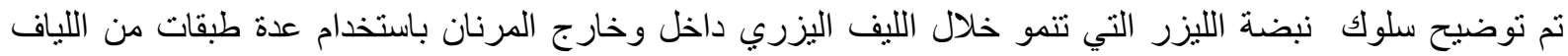

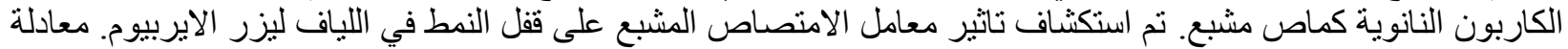

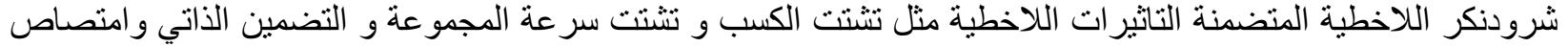

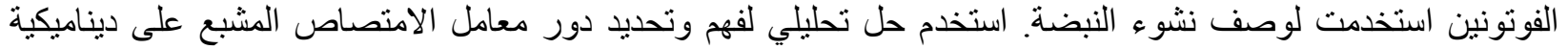

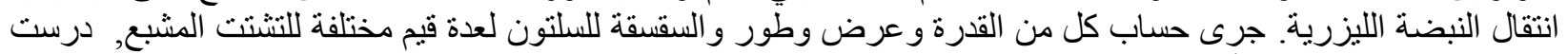
استقر ارية السلتون ضد التأثيرات اللاخطية لعدة قيم مختلفة للتشتت المشبع.

\section{Introduction}

Carbon nanotubes emerged as a promising technology for fabrication of saturable absorbers [1]. Carbon- based materials of graphene [2-4] and nanoscale graphite[5,6] have been widely investigated in a fiber ring cavity as a saturable absorber (SA) for Femto-second/ picosecond pulse generation, we can define the Graphene is an atomic layer of conjugated $\mathrm{sp} 2$ carbon atoms arranged in a two dimensional hexagonal lattice, charge carriers in it move at ultrafast speed behaving like relativistic, massless Dirac particles [7,8] Single-wall carbon nanotubes (SWNTs) have attracted much attention in the field of optical communications in recent years, due to their 
ultrafast nonlinear optical properties in the near IR arising from saturation of excitonic transitions [9-12]. exhibit interesting electronic and optical properties and have found several applications in Photonics which include nanometre-scale devices for light generation, photo-detection and photovoltaic devices $[13,14]$. Bao et al. have studied that reducing the graphene layer number can enhance the mode-locking force of graphene saturable absorber, as attributed to the enlarged modulation depth and decreased linear absorbance[15]. Erbium fiber with Carbon nanotubes (as saturable absorption and mode locking) are used to generating short optical pulses at a $22 \mathrm{MHz}$ repetition rate with $50 \mathrm{~kW}$ peak power, 1.1 ps pulse width [16]. multilayer graphene in ytterbium-doped fiber (YDF) laser to produce laser in $1 \mu \mathrm{m}$ region pulses[17], after that a few layers of graphene micro-sheets as the SA and constructed an all-fiber passively modelocked thulium-doped fiber (TDF) ring oscillator with a central wavelength of $1953.3 \mathrm{~nm} .[18]$. Most studies in the field of fiber laser have only focused on dissipative soliton generation from a graphene oxide of 11 ps laser pulses in normal dispersion cavity[19]. Recent developments in the field of the graphene nanotube have led to a renewed interest in passively mode-locked fiber laser based on a hollow-core photonic crystal fiber filled with few-layered graphene oxide solution to obtained $4.85 \mathrm{~ns}$ pulses at a $7.68 \mathrm{MHz}$ repetition rate[20]. Reduce of laser pulse is an increasingly important area in applied linguistics where a mode-locked $1.91 \mathrm{~m}$ fiber laser based on interaction between graphene oxide and evanescent field to realized $\sim 1.3 \mathrm{ps,} \sim 15.9$ $\mathrm{MHz}$ pulse laser[21]. In recent years, there has been an increasing interest in a graphene nanotube as saturable absorbers in fiber laser, where most optical systems contain graphene nanotube as saturable absorbers amplifiers with a band-limited gain. Some important examples include erbium-doped fiber laser, mode-locked lasers, and optoelectronic oscillators. The nonlinear Schrödinger equation arises in several areas of Physics, such as Optics or Quantum Mechanics, the nonlinear Schrödinger equationthat is used to model the system will have a term nonlinear, due to the gain saturation, Kerr effects, gain dispersion, and group velocity dispersion, due to the finite bandwidth of the fiber laser. In solving problems, there are mainly two types of techniques: analytic, and numerical. Analytically methods are the more rigorous ones, providing exact solutions, but they become hard to use for complex problems. A considerable amount of literature has been published on the exact solutions to nonlinear wave equations. These studies the Backlund transform [22], hyperbolic tangent expansion method [23], the trial function method [24], the nonlinear transform method [25], transformed rational function method [26] and Exact 1-soliton solutions of complex modified $\mathrm{KdV}$ equation with variable coefficients using solitary wave ansat [27].

Numerical methods as Split-Step Fourier Method [14] have become popular with the development of the computing capabilities, and although they give approximate solutions for the nonlinear Schrodinger (NLS) equation.

In this paper we shall describe and analyses way for solving the nonlinear Schrodinger (NLS) equation involves the nonlinear effects as gain dispersion, anomalous group velocity dispersion GVD and self phase modulation SPM and two photon absorption. The gain in (NLS)equation describe by the saturation power of the gain medium, the average small-signal gain, and the average power over the cavity length L. The key research question of this study was thus whether or not the short pulse change with 
varying parameter of a saturable absorption (SA), Four works will be examined, all of which focus on the relation between the power, width, phase and chirp of the pulse laser and a parameter of saturable absorption.

\section{Methodology}

In this section we describe of optical pulse propagation in a fiber laser employs the nonlinear Schrodinger (NLS) equation, and satisfied by the pulse envelope $\psi(z, T)$ in the presence of mode locking by use of a saturable absorber (no electronics are required),gain dispersion, the losses for cavity and fiber, gain, group velocity dispersion GVD and self phase modulation SPM andtwo-photon absorption optical The amplitude $\psi(z, T)$ can be assumed real if the laser frequency $\omega$ coincides with the atomic transition frequency $\omega_{0}$, this equation can be written as [5] :

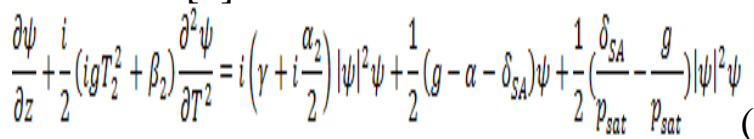

$z$ is the propagation distance, $\alpha$ takes into account material losses in the cavity, $\delta_{S A}$ saturable absorber parameter. $\alpha_{2}$ two-photon absorption parameter, $\gamma$ self phase modulation parameter, $\beta_{2}$ is the second order dispersion coefficient.In doped fibers, the gain medium responds on a time scale much slower than that of the pulse width and realized by pumping the dopants. As a result, the saturated gain may be approximated as $g=g_{0}\left(1+p_{\text {ave }} / p_{\text {sat }}\right)$ where $p_{\text {sat }}$ is the saturation power of the gain medium, $g_{0}$ is the average small-signal gain, and $p_{\text {ave }}$ represents the average power over the cavity length $\mathrm{L}, g T_{2}^{2}$ is a frequencydependent gain dispersion factor. Assume a chirped input pulse with "sech"shape. The solution corresponding to a fundamental soliton is given by:

$\psi(z, T)=\chi(z, T)+i \mu(z, T)$,

where

$\chi(z, T)=\xi \operatorname{sech}(\sigma T)(\cos (k z-\operatorname{clog}(\cosh (\sigma T))$,

and

$\mu(z, T)=\xi \operatorname{sech}(\sigma T)(\sin (k z-c \log (\cosh (\sigma T))$,

where $\xi, \sigma, k$, and $c$ are four arbitrary parameters representing amplitude, width, wave number, and chirp of the soliton, respectively.

By calculating ( $\partial \psi / \partial \mathrm{z}$ ) using Eq. (2) is found to satisfy:

$\frac{\partial \psi(z, T)}{\partial z}=\frac{\partial \chi(z, T)}{\partial z}+i \frac{\partial \mu(z, T)}{\partial z}$

The first part of Eq.(5) is given by:

$\frac{\partial \chi(z, T)}{\partial z}=-k \xi \operatorname{sech}(\sigma T)(\sin (k z-\operatorname{clog}(\cosh (\sigma T))$

The second part of Eq.(5) is given by:

$\frac{\partial \mu(z, T)}{\partial z}=k \xi \operatorname{sech}(\sigma T)(\cos (k z-\operatorname{cog}(\cosh (\sigma T))$

We can write expression for the second derivative of Eq. (2) with respect toT, after some algebra as shown in the flowing steps :

$\frac{\partial \chi(z, T)}{\partial T}=-(\chi(z, T) c+\mu(z, T)) \sigma \tanh (\sigma T)$

Let assume the new parameters as:

$\Omega=\chi(z, T) \operatorname{cotanh}(\sigma T), \quad \Gamma=\mu(z, T) \sigma \tanh (\sigma T)$,

$\frac{\partial^{2} \chi(z, T)}{\partial T^{2}}=-\left(\frac{\partial \Omega}{\partial T}+\frac{\partial \Gamma}{\partial T}\right)$,

By calculating $(\partial \boldsymbol{\Gamma} / \partial \mathrm{T})$ is found to satisfy:

$\left.\frac{\partial \Gamma}{\partial T}=-\sigma^{2} c \mu \tanh ^{2}(\sigma T)-\sigma^{2} \chi(z, T) \operatorname{sech}^{2}(\sigma T)+\sigma^{2} \chi(z, T)\right) \tanh ^{2}(\sigma T)$,

By calculating ( $\partial \Omega / \partial \mathrm{T}$ ) is found to satisfy:

$\left.\frac{\partial \Omega}{\partial T}=-\sigma^{2} c u(z, T) \operatorname{sech}^{2}(\sigma T)+\sigma^{2} c^{2} \chi(2, T) \tanh ^{2}(\sigma T)+\sigma^{2} c u(z, T)\right) \tanh ^{2}(\sigma T)$,

Substituting Eqs. (11) and (12) into Eq. (10),we get: 


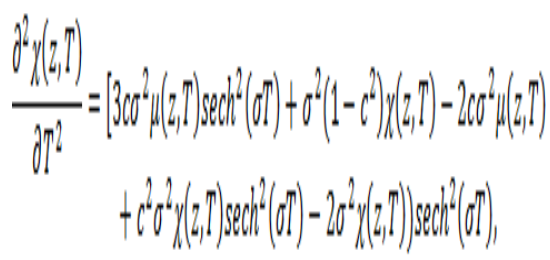

We follow a procedure similar in the above equations for finding the second derivative of Eq. (13)

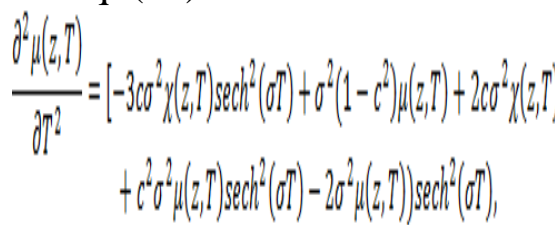

Substituting Eqs. (2), (3), (4), (7), (13) and (14) into Eq. (1), after some algebra, we obtain the following equation.

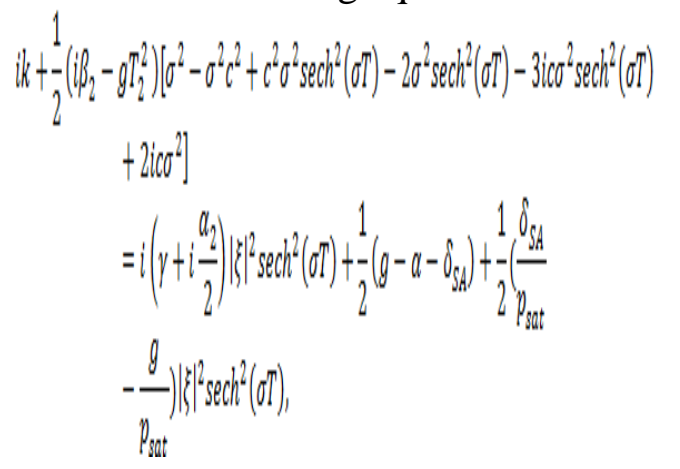

Separating the real and imaginary parts of Eq.(15), we obtain the following two equations as:
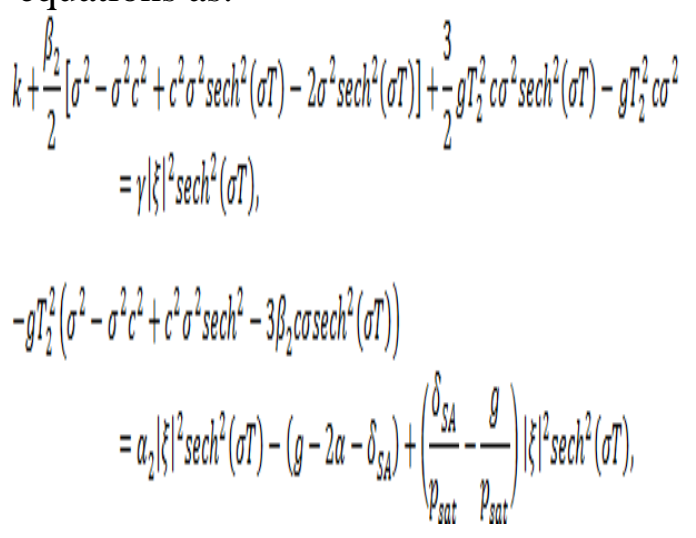

Equating the coefficient of $\sec h^{2}(\sigma \tau)$ and other parameters of Eq. (16) we obtain the following equations: $k+\frac{1}{2} \beta_{2}\left(\sigma^{2}-\sigma^{2} c^{2}\right)-g T_{2}^{2} c \sigma^{2}=013$

$\frac{1}{2} \beta_{2}\left(\sigma^{2} c^{2}-2 \sigma^{2}\right)+\frac{3}{2} g T_{2}^{2} c \sigma^{2}=\gamma|\xi|^{2}$

Eq. (17) can be separate into two equations in the form:

$g T_{2}^{2}\left(\sigma^{2}-\sigma^{2} c^{2}\right)+2 \beta_{2} c \sigma^{2}+\left(g-2 \alpha-\delta_{S A}\right)=0$

$g T_{2}^{2}\left(\sigma^{2} c^{2}-2 \sigma^{2}\right)-3 \beta_{2} c \sigma^{2}=\left(\alpha_{2}+\frac{\delta_{S A}}{p_{\text {sat }}}-\frac{g}{p_{\text {sat }}}\right)|\xi|^{2}$

\section{Results and discussion}

Laser configuration constituting a ring cavity, the detail parameters and the basic laser operation are discussed in the Methods. There are only two types of fibers used in the cavity: A piece of $6 \mathrm{~m}$ erbium-doped fiber (EDF) with negative group velocity dispersion (GVD): $-0.012 \mathrm{ps}^{2} / \mathrm{m}$ and single mode fiber (SMF) with $-0.022 \mathrm{ps}^{2} / \mathrm{m}$ GVD.

Solitonic sidebands could be observed on the spectra of the mode locked pulses, demonstrating that the net cavity dispersion is anomalous in the present cavity. A $10 \%$ fiber coupler was used to output the laser. The laser is counter-directionally pumped by a $980 \mathrm{~nm}$ laser diode; A16polarization independent isolator was spliced in the cavity to force the unidirectional operation of the ring. An intra cavity polarization controller was used to change the cavity linear birefringence. Graphene mode-locker was placed between the output couple and polarization controller as shown in Fig.1.

\section{Chirped soliton}

Eqs. (19) and (21) can be used to find a equation for chirp of the soliton 


$$
\begin{gathered}
{\left[2 \gamma g T_{2}^{2}-\left(\alpha_{2}+\frac{\delta_{\text {SA }}}{p_{\text {sat }}}-\frac{g}{p_{\text {sat }}}\right) \beta_{2}\right] c^{2}-3\left[2 \gamma \beta_{2}+\frac{1}{2} g T_{2}^{2}\left(\alpha_{2}+\frac{\delta_{S A}}{p_{\text {sat }}}-\frac{g}{p_{\text {sat }}}\right)\right] c} \\
-2\left[2 \gamma g T_{2}^{2}-\left(\alpha_{2}+\frac{\delta_{\text {SA }}}{p_{\text {sat }}}-\frac{g}{p_{\text {sat }}}\right) \beta_{2}\right]=0
\end{gathered}
$$

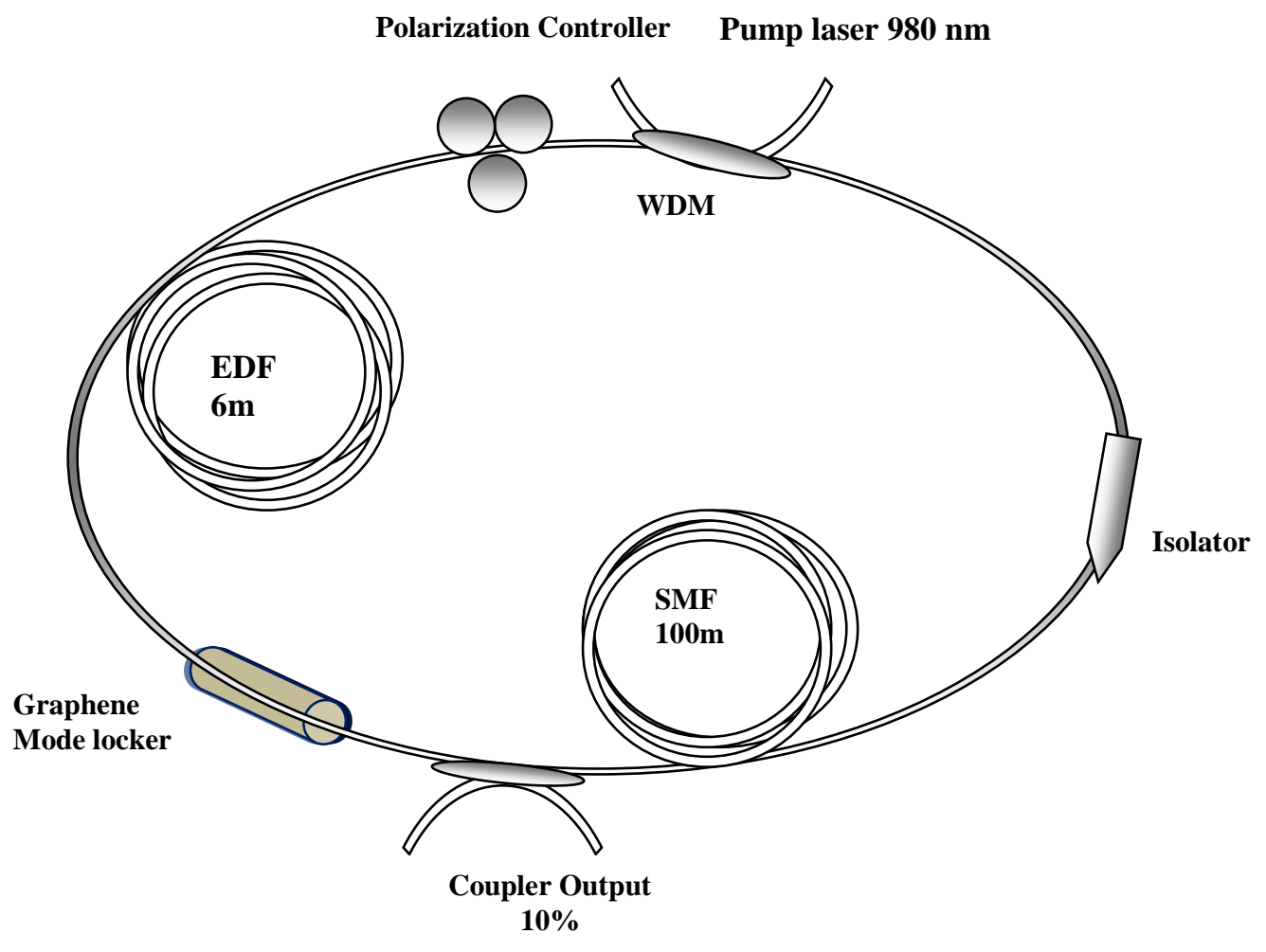

Fig . 1: This schematic shows the fiber laser components such as erbium doped fiber (EDF), single mode fiber (SMF), wavelength division multiplexer (WDM), polarization controller, coupler and optical isolator.

After some algebra, we obtain the following equation

$$
c^{2}-3 \frac{\left[2 \gamma \beta_{2}+\frac{1}{2} g T_{2}^{2}\left(\alpha_{2}+\frac{\delta_{\text {SA }}}{p_{\text {sat }}}-\frac{g}{p_{\text {sat }}}\right) \beta_{2}\right]}{\left[2 \gamma g T_{2}^{2}-\left(\alpha_{2}+\frac{\delta_{\text {SA }}}{p_{\text {sat }}}-\frac{g}{p_{\text {sat }}}\right) \beta_{2}\right]} c-2=0 .
$$

We can rewrite above equation as:

$$
c^{2}-m c-n=0
$$

where the parameter $m$ and $n$ given by:

$$
m=-3\left(\frac{\left[3 \gamma \beta_{2}+\frac{1}{2} g T_{2}^{2}\left(\alpha_{2}+\frac{\delta_{S A}}{p_{\text {sat }}}-\frac{g}{p_{\text {sat }}}\right) \beta_{2}\right]}{\left[2 \gamma g T_{2}^{2}-\left(\alpha_{2}+\frac{\delta_{\text {SA }}}{p_{\text {sat }}}-\frac{g}{p_{\text {sat }}}\right) \beta_{2}\right]}\right), \quad n=-2
$$


It is easy to show that the chrip parameter is found to satisfy:

$c=\frac{m}{2} \pm \sqrt{\frac{m^{2}-4 n}{4}}$

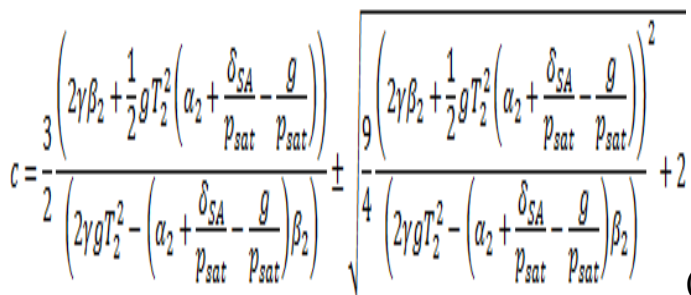

To understand the dynamical behavior of the chirp, we must investigate of Eq. (27)

We have three cases

Case1: The solution Eq.(27) is called the symmetric state and exists only for $m^{2}-4 n>0$ the chirp parameter is realand indicates that the corresponding soliton propagate without changes in its shape, width, or amplitude and the soliton is stable.

Case 2: when the value $m^{2}-4 n=0$ and the chirp value given by $c=m / 2$, this is indicates that the frequency is shifted (chirp) always take place for soliton laser.

Case 3: The solution Eq.(27) represents an anti-symmetric state and exists for all $m^{2}-4 n>0$. In these cases, the chirp parameter is imaginary and it is not possible to obtain the soliton because it is purely imaginary and the anti-symmetric states are unstable. From the condition of the real value of a chirp parameter, we get this equation

$\frac{9}{4}\left(\frac{2 \gamma \beta_{2}+\frac{1}{2} g T_{2}^{2}\left(\alpha_{2}+\frac{\delta_{\text {SA }}}{p_{\text {Sat }}}-\frac{g}{p_{\text {Sat }}}\right)}{2 \gamma g T_{2}^{2}-\left(\alpha_{2}+\frac{\delta_{\text {SA }}}{p_{\text {sat }}}-\frac{g}{p_{\text {Sat }}}\right) \beta_{2}}\right)^{2}+2 \geq 0$

The saturable absorption parameter according to Eq. (28) given with this condition :
$\delta_{S A} \geq \frac{\left(i \frac{4}{3} \sqrt{2} \gamma-\frac{a_{2}}{2}+\frac{g}{2 p_{\text {sat }}}\right)}{\left(\frac{g T_{2}^{2}}{2 p_{\text {sat }}}+\mathrm{i} \frac{2}{3} \sqrt{2} \frac{\beta_{2}}{p_{\text {sat }}}\right)} g T_{2}^{2}-\frac{\left(i \frac{2}{3} \sqrt{2}\left(a_{2}+\frac{g}{p_{\text {sat }}}\right)+2 \gamma\right)}{\left(\frac{g T_{2}^{2}}{2 p_{\text {sat }}}+\mathrm{i} \frac{2}{3} \sqrt{2} \frac{\beta_{2}}{p_{\text {sat }}}\right)} \beta_{2}$

The saturable absorption parameter $\delta_{S A}$ was related to the imaginary part of $4 / 3 \sqrt{2} \gamma, 2 / 3 \sqrt{2} \beta_{2} / p_{\text {sat }}$ and $2 \sqrt{2} / 3\left(\alpha_{2}+g / p_{\text {sat }}\right)$ since the light was propagated in the forward direction. In a fiber laser with a saturable absorption mode locker, the saturable absorption parameter $\delta_{S A}$ depend on the $g T_{2}^{2}$, $\beta_{2}, \alpha_{2}, g / 2 p_{\text {sat }}$, and $\gamma$.

We can use Eq. (27) to calculate the chirp of the soliton for values of $\delta_{S A}$ in the range from 0.1 to 0.4 . Fig. 2 shows changes in the chirpas a function of the saturableabsorption parameter $\delta_{S A}$, and also shows that the chirp increases linearly with the saturable absorption parameter $\delta_{S A}$.

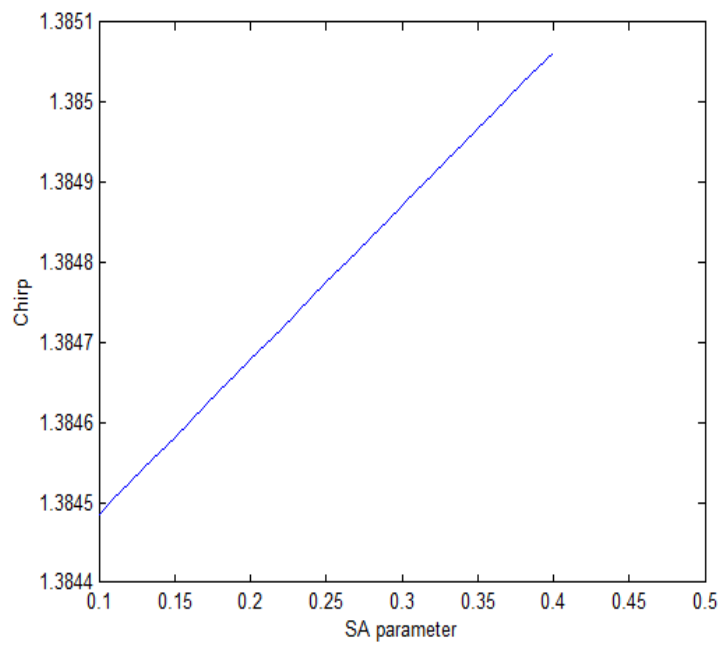

Fig.2: Chirp versus saturable absorption parameter $\delta_{S A}$.

Graphene has two comporents to its saturable absorption: one fast, the other slow [4]. The time constant for the slow component is approximately 1.1 picoseconds. The fast component is not resolved due to the long duration of the pulses measuring the saturable absorption ( 210fs). Eq. (30) describes this behavior of $T_{F W H M}$ as a function to fiber laser parameters. 
$\delta_{S A}=\frac{1}{T_{F W H M}^{2}}\left(2 \beta_{2} c+g T_{2}^{2}-g T_{2}^{2} c^{2}\right)+(g-2 a)$

Fig.3(a) shows the soliton width (FWHM) as a function of $\delta_{\text {SA }}$. The soliton width increases exponentially with $\delta_{S A}$, The increase saturable absorption mode locker $\delta_{S A}$ reduce the power of the soliton Eq. (31) shows that $\delta_{S A}$ depend on the saturable power of graphene $p_{\text {sat }}$ and the

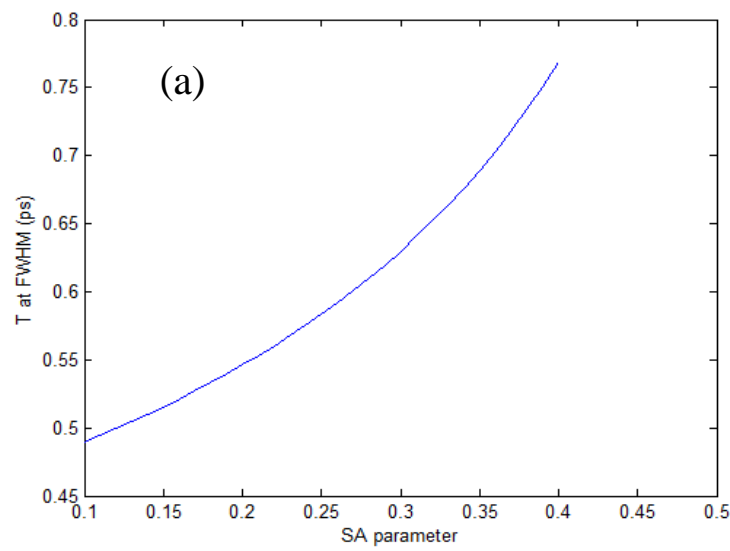

power of the soliton $p$ as sBOwn in and Fig.3(b). The building pulse evolves toward such a soliton by reducing its width and increasing its peak power.

$\delta_{S A}=\frac{p_{\text {sat }}}{p} \frac{T_{2}^{2}}{T_{\text {FWHM }}^{2}} g\left(c^{2}-2\right)-3 \frac{p_{\text {sat }}}{p} \frac{\beta_{2}}{T_{\text {FWHM }}^{2}} c+\left(g-a_{2} P_{\text {Sat }}\right)$

Fig.3(a): Output powers, (b)widths of soliton for different values of $\delta_{S A}$.

Fig.4(a) the saturable absorption of graphene near the Dirac point a photon with energy $E=h f$ excites an electron from the valence band to the conduction band. Fig.4(b) electrons excited into the conduction band lose energy and momentum to the crystal lattice and to electron-electron scattering; the holes they left behind behave similarly. This allows new electrons to be excited to this same energy level and contributes to intraband relaxation time of the absorber. Fig.4(c) at high enough peak intensities and pulse fluencies, enough electrons have been excited into the valence band that there are no open states of lower energy in the conduction band. Absorption and stimulated emission reach a steady state and the absorber is saturated. Fig. 4(d) shows how the saturable absorption reduces the pulse width.

\section{Unstable laser pulse}

Analytical simulations for growth pulses propagating inside a 5-m fiber ring without saturable absorption mode locker shows that each input pulse develops an internal substructure consisting of many subpulses of width fs. Figs. 5 shows the evolution toward a parabolic shape when a "sech" input pulse is amplified over the $5 \mathrm{~m}$ length of the fiber laser, the position and width of subpulses change continuously in an apparently random manner. Changing anomalous GVD values, by varying the length of SMF, therefore, the total cavity dispersion was varied [28, 29]. Figs.5 shows that each pulse still develops an internal substructure in which depending on frequencies of the photons laser through the ring resonator, the substructure varies from pulse to pulse and exhibits chaos, and the pulse power also varies from pulse to pulse and exhibits chaos behavior as phase is varied in the cavity resonance. 
(a)

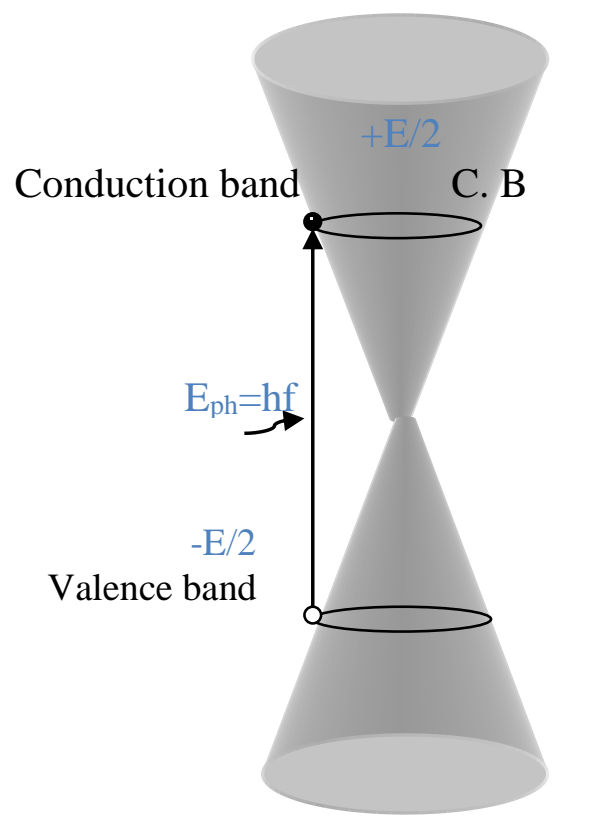

(b)

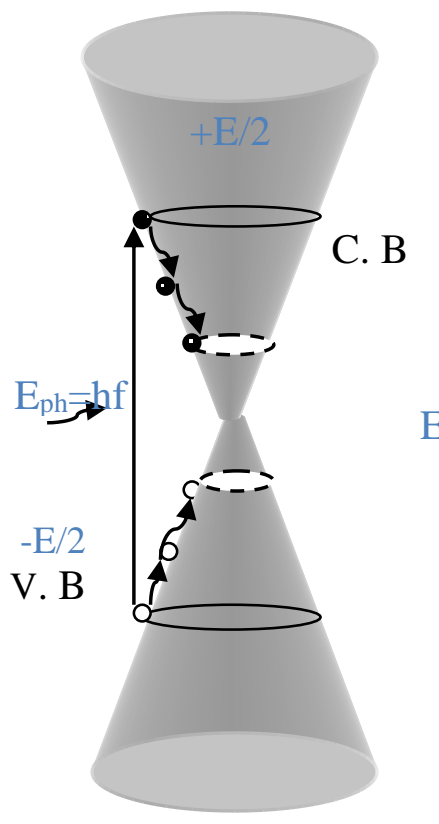

(c)

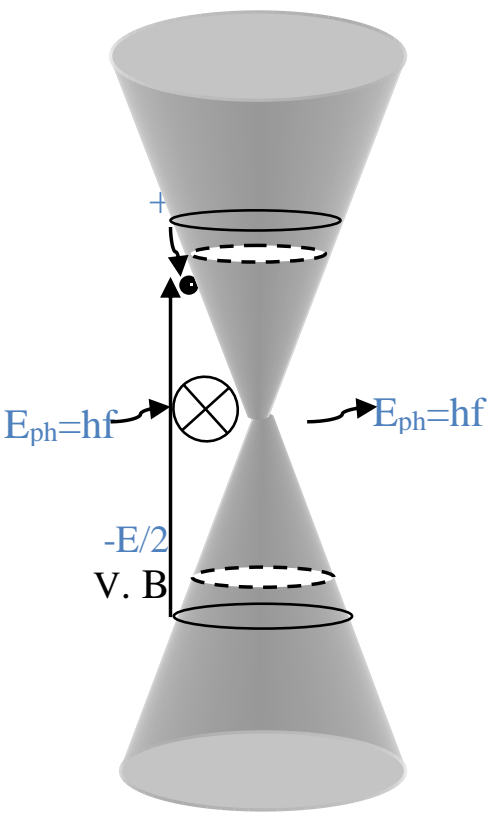

(d)

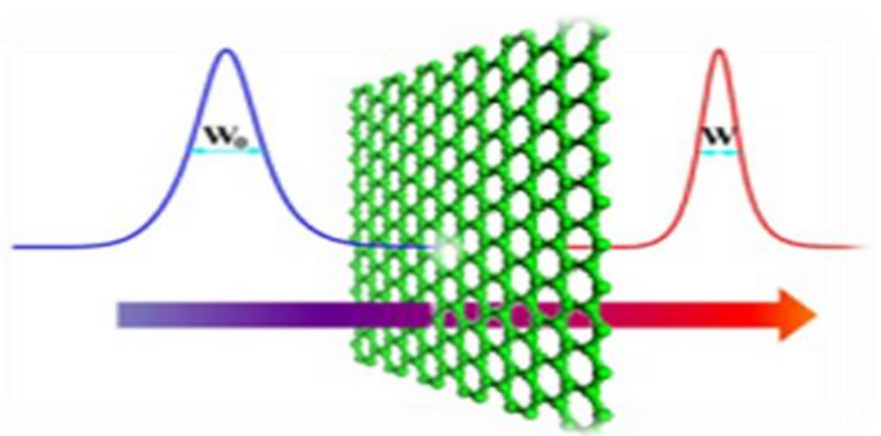

Fig.4: The mechanism of saturable absorption mode locker.

\section{Stable pulse (soliton)}

The real solution Eq.(3) provides a shortcut to understanding the behavior pulse laser propagation through $\mathrm{Er}^{+3}$ ring cavity length $6 \mathrm{~m}$ with saturable absorption mode locker. In this section, we will focus on the effects of saturable absorption parameter on the behavior of the laser pulse, the saturable absorption parameters are designed to be tuned in a wide range from 0.1 to 0.4 by varying the thickness of graphene. When the $\delta_{S A}=0.1$ and using Eqs. (3), (18), (19), (20) and (27), solitons have been formed in a $6 \mathrm{~m}-$ long EDFL. Figs. (6) and (7) show pulse shapes at the output endof the saturable absorption mode locker when the parameters system are $\gamma=0.012(\mathrm{Wm})^{-1}, \beta_{2}=-0.014 \mathrm{ps}^{2} / \mathrm{m}$, $\alpha_{2}=0.3, \quad \mathrm{~T}_{2}=0.047 \mathrm{ps}, \quad \alpha=0.1782 \mathrm{~m}^{-1}$, $p_{\text {sat }}=210 \mathrm{~W}$ and $g=0.7827 \mathrm{~m}^{-130}$.

The power of the soliton grows exponentially once the power of pulse exceeds a threshold value, where at $z=10 \mathrm{~cm}$ the power of soliton be at 0.007 $\mathrm{W}$, and picosecond optical pulse is amplified in such EDFL, until gain saturates at $z=4 m$ with power $1.2 \mathrm{~W}$. 

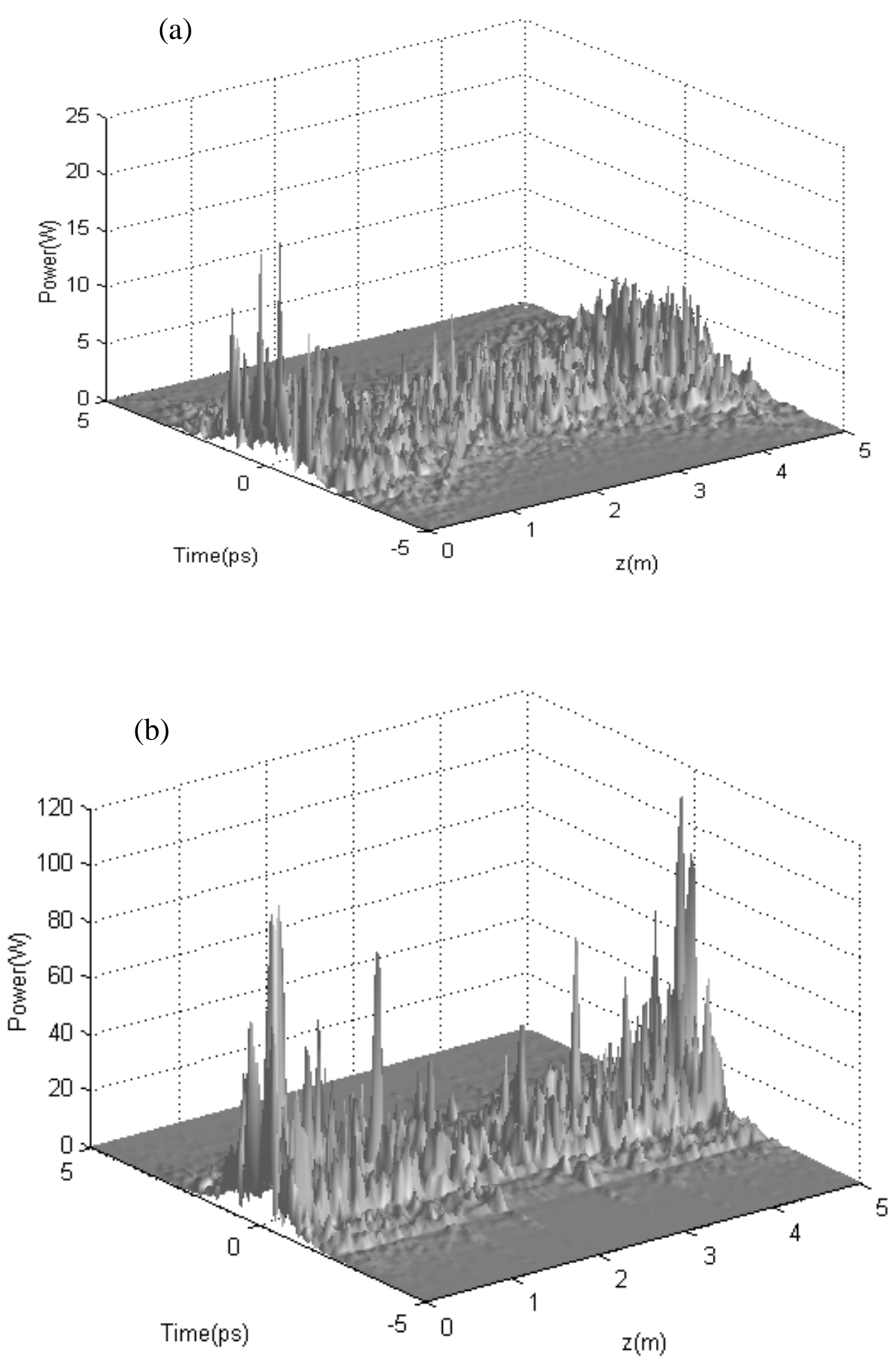

Fig.5: Evolution of substructure in a 5-m ring resonator when pulses growth from spontaneous emission, with $G V D$ (a) $\beta_{2}=-0.014 \mathrm{ps}^{2} / m$ (b) $\beta_{2}=-0.012 \mathrm{ps}^{2} / m$ and parameters are $\gamma=0.012(\mathrm{Wm})^{-1}$, $\alpha_{2}=0.3, T_{2}=0.047 \mathrm{ps}, \alpha=0.1782 \mathrm{~m}^{-1}$ and $g=0.7827 \mathrm{~m}^{-1}$. 

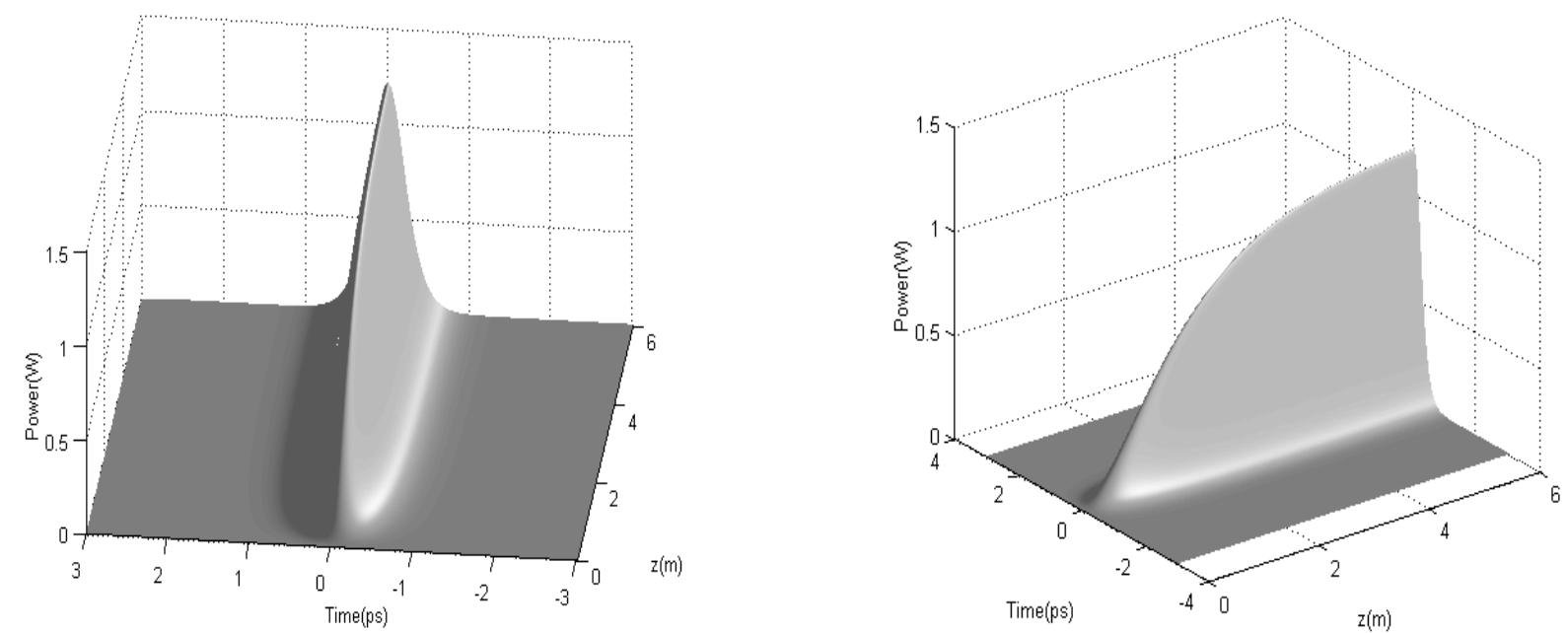

Fig.6: Soliton growth from spontaneous emission with EDFL parameters are $\gamma=0.012(W m)^{-1}$, $\beta_{2}=-012 \mathrm{ps}^{2} / \mathrm{m}, \alpha_{2}=0.3, T_{2}=0.047 \mathrm{ps}, \alpha=0.17822771 \mathrm{~m}^{-1}, g=0.7827525 \mathrm{~m}^{1-}, p_{\text {sat }}=210 \mathrm{~W}$, and $\delta_{S A}=0.1$.
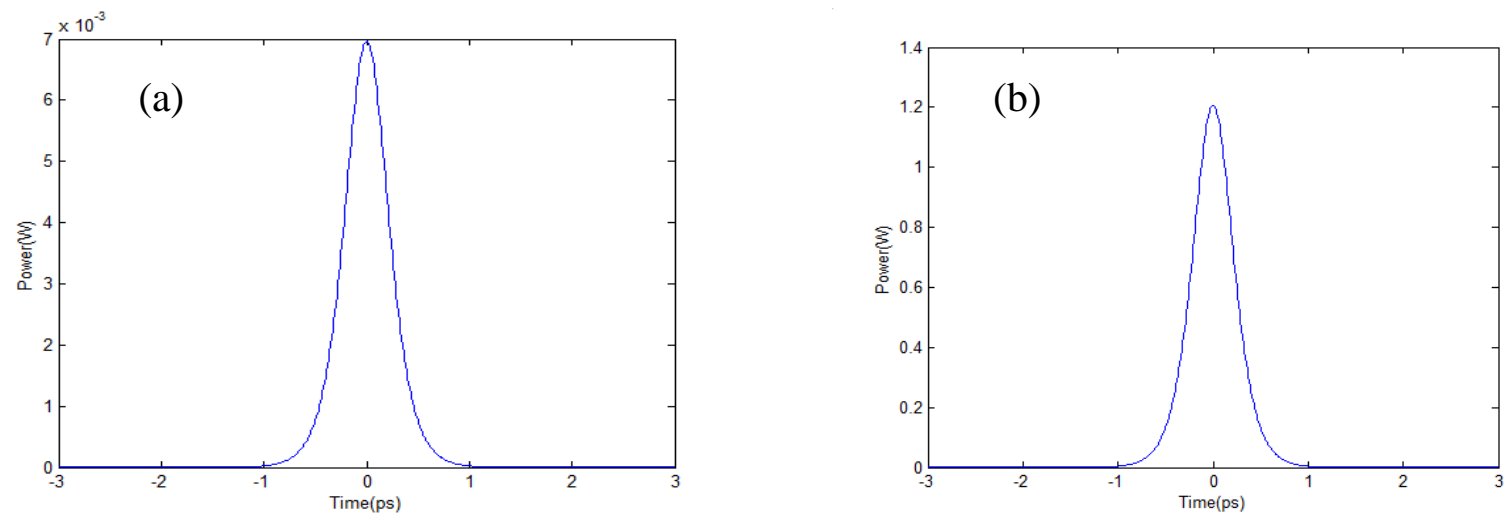

Fig.7: The profiles of the parabolic pulse at (a) $z=0.1 \mathrm{~m}$ (b) $z=6 \mathrm{~m}$.
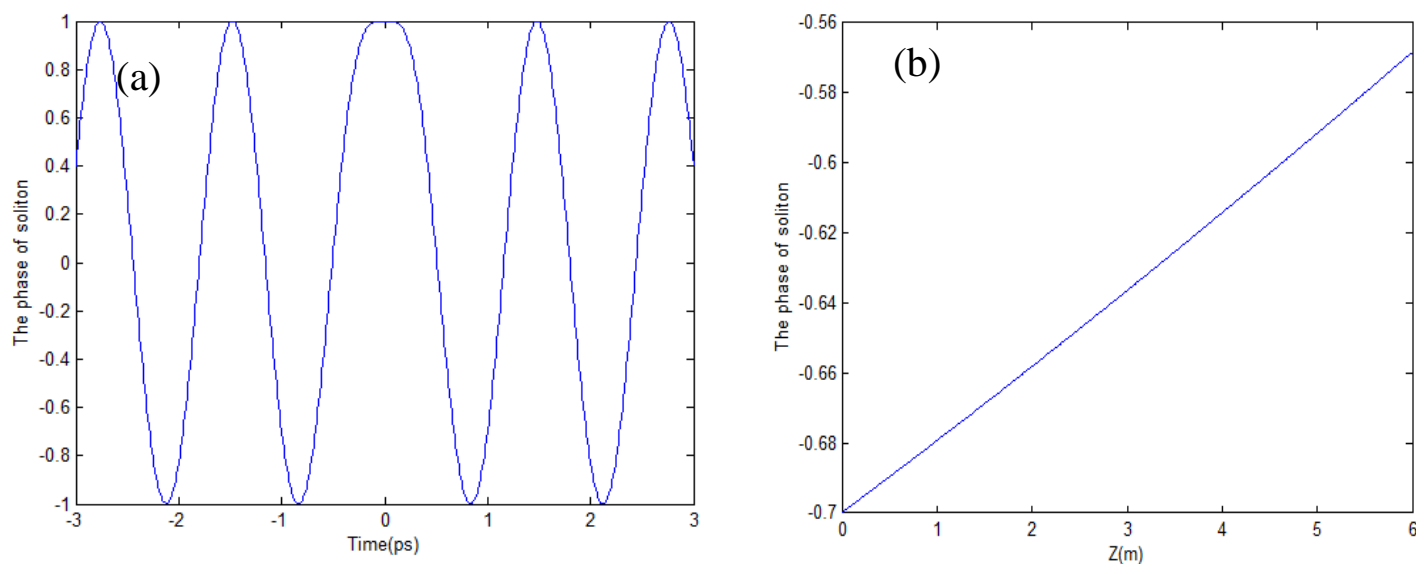

Fig. 8: The phase of soliton plotted as a function of (a) time (b)fiber length.

Fig.8 shows the variation of the soliton phase (a) with time (b) with the length of fiber laser according to equation of phase $\varphi=k z-\operatorname{clog}(\cosh (\sigma T)$, when fixed the 
length at $z=4 m$,we see that the phase changing with time as cosine wave with rang 1 to -1as shown in Fig.8(a). The wave number in phase equation depends on the parameters $\beta_{2}, g T_{2}^{2}, \mathrm{c}$ and width of soliton $T_{F W H M}$ as shown in the following equation :

$k=\frac{1}{2 T_{F W H M}^{2}}\left(\beta_{2} c^{2}+2 g T_{2}^{2} c-\beta_{2}\right)$

let us consider the case in which the phase of soliton varies linearly along the fiber length as shown in Fig.8(b) at $z=0 m$ the phase of soliton $\varphi=-0.7$, where $\varphi=-\operatorname{clog}(\cosh (\sigma T)$ and the phase of soliton reached maximum value at $\varphi=-0.57$. It is clearthat the values of the phase will play an important role to building soliton from spontaneous emission in $\mathrm{Er}^{+3}$ fiber laser.

\section{Effects of changing SA on behaviour of soliton}

Figs.9(a) and 9(b) show the effect of changing saturable absorption mode locker $\delta_{S A}$ on the shape of soliton the evolution toward the spectral sidebands generated through a self phase modulation (SPM)affects, anomalous group velocity GVD effects, gain dispersion do not grow with the propagation of solitons. The power of soliton grows exponentially, along the fiber length; the soliton is propagated around the laser cavity repeatedly until a steady state is reached. Saturable absorber can also be used to filter sidebands associated with solitons since sidebands can be transferred selectively to thelossy core because of its low power level.

The power of the soliton infiber resonators is evident from Eq.(33): Note that the power depends on $\operatorname{GVD}\left(\beta_{2}\right), \quad$ gain dispersion $\left(g T_{2}^{2}\right), \operatorname{chirp}(\mathrm{c}), \operatorname{SPM}(\gamma)$, and width of soliton $T_{F W H M}$.

$|\xi|^{2}=\frac{1}{2 \gamma T^{2}{ }_{F W H M}}\left(-\beta_{2} c^{2}+3 g T_{2}^{2} c+\beta_{2}\right)$
$T_{F W H M}=1.66 \sqrt{\frac{-g T_{2}^{2}\left(1-c^{2}\right)-2 \beta_{2} c}{\left(g-2 \alpha-\delta_{S A}\right)}}$

It is evident from Eqs. (33) and (34) that the parameters $\xi$ and $T_{F W H M}$ can have real positive values and imaginary values, where these values plays an important 3 irole to stable soliton exists only if $\xi$ and $T_{F W H M}$ are real. This feature can alsobe understood from Figs.10(a) and 10(b) show that the pulse with a saturable absorption mode locker form a soliton and becomes broader with increasing the values of $\delta_{S A}$.
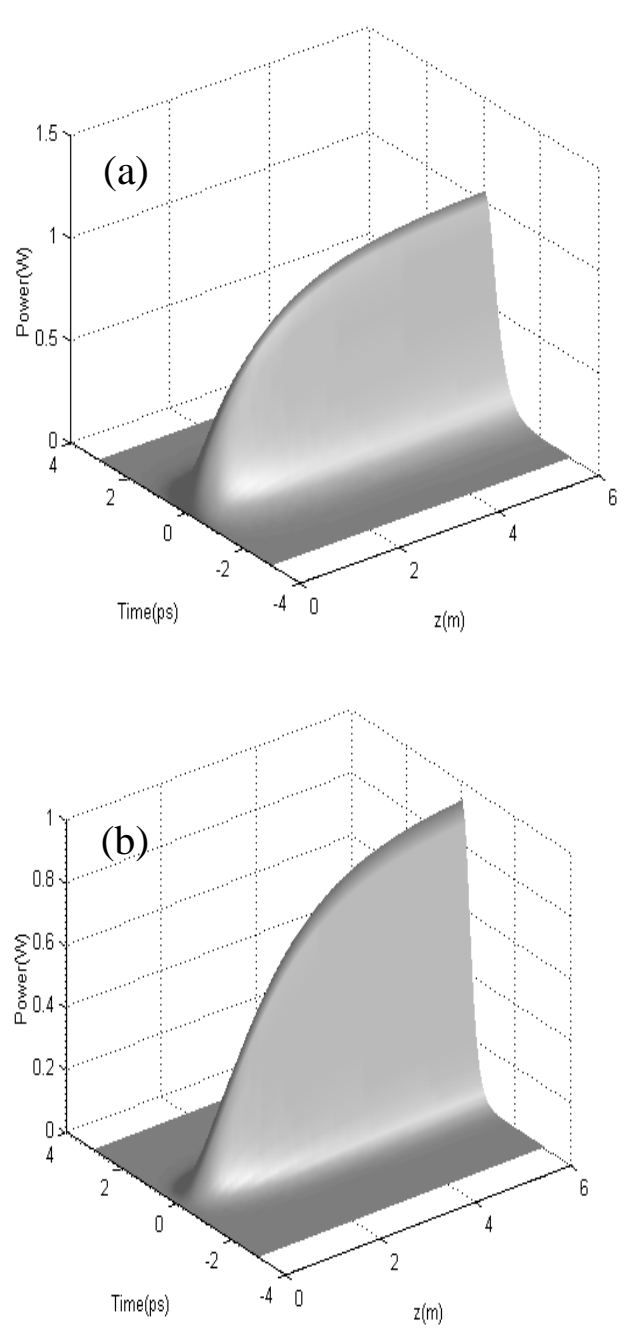

Fig.9: Soliton growth from ${ }^{53}$ spontaneous emission $(a) \delta_{S A}=0.2(b) \delta_{S A}=0.3$. 

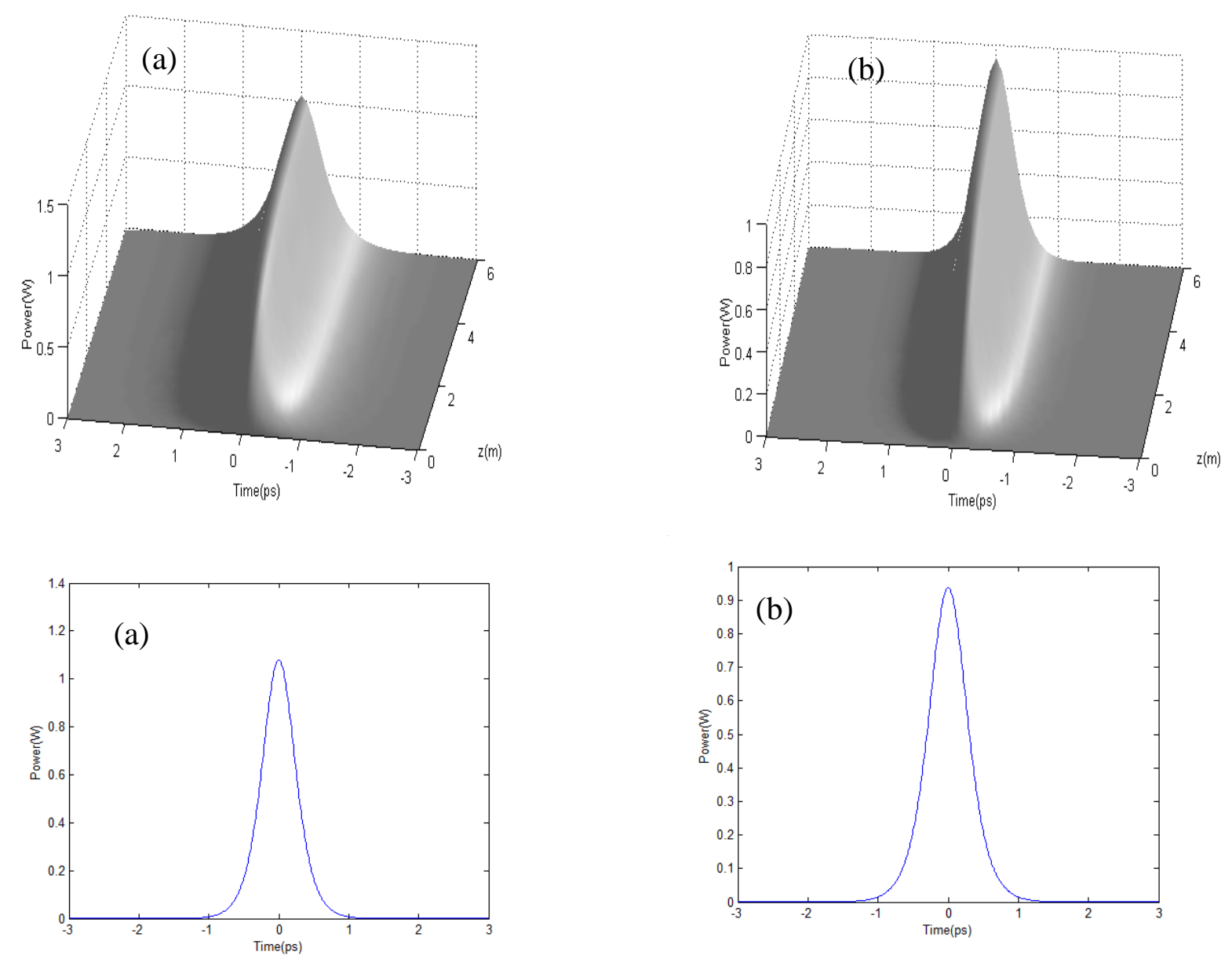

Fig.10: Soliton width for $(a) \delta_{S A}=0.2(b) \delta_{S A}=0.3$.

\section{Conclusions}

We highlight four important aspects related to a mathematical model of pulsed fiber lasers with ring cavity a power, width, chirp and phase of the soliton on generation of single optical pulses. Picosecond laser pulse at power $\sim 1.2 \mathrm{~W}$, width $\sim 0.5$ ps can be generated using monolayer graphene as saturable absorber and form soliton, the power, width, phase, the chirp of laser soliton vary by changing the intracavity loss with resonator length $6 \mathrm{~m}$. Our results in regards to the parabolic shape of the pulse and the behavior of the laser pulse have good agreement with the results in the references[30 -36].

\section{References}

[1] S. Y. Set, H. Yaguchi, Y. Tanaka, and M. Jablonski, J. Lightwave Technol. 22, 1 (2004) 51.

[2] F. Bonaccorso, Z. Sun1, T. Hasan1 \& A. C. Ferrari., Nat.Photonics, 4, 9 (2010) 611622.

[3] G. Sobon, J. Sotor, and K. M. Abramski, Appl. Phys. Lett. 100, 16 (2012) 161109161112.

[4] Q. Bao and K. P. Loh, ACS Nano, 6, 5(2012) $3677-3694$.

[5] G. R. Lin and Y. C. Lin, Laser Phys. Lett. 8,12 (2011) 880-886.

[6] Y. H. Lin and G. R. Lin, Laser Phys. Lett. 9, 5 (2012) 398-404. 
[7] K. S. Novoselov, A. K. Geim, S. V. Morozov, D. Jiang, Y. Zhang, S. V. Dubonos, I. V. Grigorieva, A. A. Firsov, Science, 306 (2004) 666-669.

[8] K. S. Novoselov, A. K. Geim, S. V. Morozov, D. Jiang, M. I. Katsnelson, I. V. Grigorieva, S. V. Dubonos, A. A. Firsov, Nature, 438 (2005)197-200.

[9] Y.C. Chen, N.R. Raravikar, L.S. Schadler, P.M. Ajayan, Y.P. Zhao, T.M. Lu, G.C. Wang, X.C. Zhang,, Appl. Phys. Lett. 81(2002) 975.

[10] T. Hertel, R. Fasel, and G. Moos, Appl. Phys. A 75 (2002) 449.

[11] S. Tatsuura, M. Furuki, Y. Sato, I. Iwasa, M. Tian, and H. Mitsu, Adv. Mater. 15 (2003) 534.

[12] J-S. Lauret, C. Voisin, G. Cassabois, C. Delalande, Ph. Roussignol, O. Jost, and L. Capes, Phy. Rev. Lett. 90 (2003) 057404.

[13] H. Kataura, Y. Kumazawa, Y. Maniwa, I. Umezu, S. Suzuki, Y. Ohtsuka, and Y. Achiba, Synth. Met. 103,1-3 (1999) 25552558.

[14] P. Avouris, M. Freitag, and V. Perebeinos, Nat. Photonics, 2, 6 (2008) 341350.

[15] Q. L. Bao Adv. Funct. Mater. 19,19 (2009) 3077-3083

[16] L. Antonucci, A. Jullien, O. Albert, D. Douillet, J.-P. Rousseau, and G. Cheriaux, Lasers and Electro-Optics, 1 (2007) 1.

[17] L. M. Zhao, D. Y. Tang, H. Zhang, X. $\mathrm{Wu}$, Qiaoliang Bao, and Kian Ping Loh., Opt. Lett. 35,21 (2010) 3622-3624.

[18] J. H. V. Helden, N. Lang, U. Macherius, H. Zimmermann, and J. Reopcke, Appl. Phys. Lett. 102, 13 (2013) 131114-131117.

[19] Jia Xu, Sida Wu, Huihui Li, Jiang Liu, Ruoyu Sun, Fangzhou Tan, Quan-Hong Yang, and $\mathrm{Pu}$ Wang, Opt. Express, 20, 21(2012) 23653-23658.

[20] Z. Liu, X. He, and D. N. Wang, Opt. Lett. 36, 16 (2011) 3024-3026.
[21] M. Jung et al., Appl. Phys. Express, 5, 11 (2012) 112702-112704.

[22] C. Rogers and W. F. Shadwick Backlund Transformations (New York: Cambridge University Press) (ed.) D G Crighton p 67(1982).

[23] E. M. E. Zayed, H.A. Zedan and K.A. Gepreel J. Nonlinear Sci. Numer. Simul. 5, 3 (2004) 221-234

[24] M. Inc, D. J. Evans, J. Comput. Math. 81, 2 (2004), 191-202.

[25] J. L. Hu, Phys. Lett.A322, 3-4 (2004) 211-216.

[26] W. X. Ma and J. H. Lee Chand, Chaos Solitons Fractals.42, 3 (2009) 1356-1363.

[27] H. Kumar and F, Indian Journal of Physics, 87, 9 (2013) 909-912.

[28] C. Vinegoni, M. Wegmuller, and N. Gisin, IEEE Photonics Technol. Lett. 13, 12 (2001) 1337-1339.

[29] M. Faucher, E. Villeneuve, Benoit Sevigny, Alexandre Wetter, Roger Perreault, Yannick Keith Lizé, Nigel Holehouse, Proc. SPIE6873, 68731T (2008). [30] Áron Szabó and Zoltán Várallyay, IEEE Photonics Technology Letters, 24,2 (2012) 122- 124.

[31] I.A. Yarutkina, O.V. Shtyrina, M. P.Fedoruk, S. K. Turitsyn, Opt. Express, 21,10 (2013) 12942-12950.

[32] Huiquan Li,et al, Optical Engineering. 52,12 (2013) 126102-1-4.

[33] Xin Zhao, Zheng Zheng, Lei Liu, Ya Liu, Yaxing Jiang, Xin Yang, and Jinsong Zhu, Opt. Express, 19, 2 (2011) 1168-1173.

[34] Amos Martinez, Kaiming Zhou, Ian Bennion, and Shinji Yamashita, Opt. Express,18,11(2011) 11008-11014.

[35] Qi Feng et al, Optical Engineering, 52,4 (2013) 044201-1-9

[36] Marc Currie, Travis Anderson, Virginia Wheeler, Luke O. Nyakiti, Nelson Y. Garces, Rachael L. Myers-Ward, Charles R. Eddy, Jr. Fritz J. Kub, D. Kurt Gaskill, Optical Engineering 52, 7 (2013) 0761011-5. 